\title{
The Inheritance and Development and Innovation of Zhucheng Guqin in Shandong
}

\author{
Xiaopeng Sun \\ Binzhou Vocational College \\ Huanghe Science and Technology College \\ Binzhou, China \\ e-mail: zhangwei492@163.com
}

\begin{abstract}
So far, the Zhucheng Guqin in Shandong has a history of 200 years or so. The predecessors have made some achievements in developmental research on the Zhucheng Guqin in Shandong. In the process of development, the Zhucheng Guqin has positive significance on the inheritance of folk culture and plays an important role in it. Meanwhile, with the social changes and cultural development, the Zhucheng Guqin has changed in some degree in different periods of history development. On the basis of collecting the developing situation and inheriting system of Guqin of Zhucheng, this article combines the general situation of geography humanities in Zhucheng and aims at the excellent cultural potential of the Zhucheng Guqin, putting forward the reasons of the inheritance, development and innovation of the Zhucheng Guqin and the ideas and constructive suggestions about how to develop the Zhucheng Guqin.
\end{abstract}

Keywords-Zhucheng Guqin; origin; development; inheritance

\section{THE ORIGIN OF THE ZHUCHENG GUQIN IN SHANDONG}

Zhucheng city is known as Dongwu or Mizhou in ancient times, a county-level city under the government of Weifang city, Shandong province in China. It is located in Jiaodong region of Shandong Peninsula and an important hub in Shandong Peninsula. Except for ethnic Han, there are 8 minorities such as Manchu, Hui, Tibetan, Zhuang, Yao, North Korea, Mongolia and Xibe, etc. Zhucheng city has a long history and it is the birthplace, the village of Zhufeng located in the north of the the city, of Shun Emperor, an enlightened monarch in ancient times. Zhucheng gets its name because of this. Zhucheng has rich cultural deposits and long historical and cultural atmosphere, providing deep roots and soil for the inheritance and development of a variety of folk culture.

As an independent category of musical instruments, the Zhucheng Guqin develops and spreads in rural community largely. The formation of its style has close connection with folk music culture in Shandong province and has profound influence on it. [1] The foundation of the Zhucheng Guqin in Shandong can trace back to "Yushan" and "Jinling" in the middle of 19th century. The Zhucheng Guqin has a history of over 200 years till now. It is founded by Wang Jifu (whose given name is Puchang,1807-1886) and Wang
Lengquan(whose given name is Yumen,1807-1877) and they respectively learn from Yushan and Jinling. So their playing style is different, such as the master works, The Complain at Changmen Palace and Lyrics of Autumn Wind. On the whole, as an important school of Guqins in our country, initially it can only spread in Zhucheng city and then develop gradually. In the process of its subsequent development, the representative personages are: the founder Wang Jifu, Wang Lengquan, Wang Zuozhen as inheritor of the first generation of Wang Jifu, Wang Binlu as inheritor of the first generation of Wang Lengquan, Wang Xi and Wang Lu as inheritors of the second generation of Wang Jifu. The Zhucheng Guqin has music style of Wang Jifu and Wang Lengquan, the "upright and gentle characters" of "Yushan" and the "gorgeous and lingering characters" of "Jinling". Therefore, the unique style of music of Zhucheng comes into being. [2]

\section{THE CURRENT SitUATION OF THE ZHUCHENG GUQIN IN SHANDONG}

\section{A. The development of the Zhucheng Guqin in Shandong}

In the process of development, even though the Zhucheng Guqin experiences the flourish in the late Qing Dynasty and the beginning of the Republic of China and becomes very popular at one time. It has produced prodigious influence Guqin arts and lays the foundation for the future development of the Guqin in Zhucheng. However, because of many factions derived in the subsequent development and the force of circumstances in the early time of the Great Cultural Revolution, this Guqin art suffers from difficulties inevitably. The activities such as Guqin associations and Guqin mass organization are forced to interrupt. As the older generation of Guqin artists die one after another, the Zhucheng Guqin can pass on nobody like many other folk arts, lacking successors, and for the living Guqin artists at an advanced age, the Guqin art can only be treated as entertainment or taught to some students, not forming a system. For example the transmission scale of guiqin of Zhan Qingyun and Yuan Shuheng etc is not big. Under the influence of this situation, some descendants of guiqin in Zhucheng have made great efforts to promote and explore the culture of Guqin in Zhucheng in recent years. Among them, Pang Yuzhu as disciple of Zhang Yujin uses the leisure time after he retires to focus on the study of Guqin 
music, publishing more than 50 monographs on Guqin research such as The Brief Course of Guqin and The Anthology of Chinese Guqin, which make great contributes for the excavation and inheritance of Guqin art in Zhucheng. Besides, Wang Xiaotian as the grandson of Yuan Shujun and Yuan Shuheng followed his great-grandmother Zhan Jingqiu and great uncle grandfather to learn Guqin of Zhucheng from a child and founded Deyin Guqin Club in November, 2003, providing a broad platform for the research and promotion of Guqin of Zhucheng. They have held seminars for many times and trained more than 50 disciples, among whom more than 40 people took part in the national level examination of Guqin, and more than 20 people won prizes in folk music competition in province and city and many people were selected into national championship or entered the conservatory of music of national level. In December, 2004, Deyin Guqin Club undertook the folk music view and emulate concert for national juvenile; in July, 2005, Guqin Professional Committee of Society of Ethnic Orchestral Music in Jinan City was approved to set up. The notable success of Deyin Guqin Club attracts music lovers from many places in Shandong, creating a new situation for the promotion of Guqin art in Zhucheng.

Except for the Zhucheng Guqin art lovers active in Shandong region, the branch of Wang Yanqing as the descendants of later generations of Wang Lengquan is valued by reformers leader Kang Youwei in the reform period of Qing dynasty. Wang Yanqing was recommended to teach in "Meian Campus" in Nanjing Higher Normal School for 10 years. The disciples who have more influence such as $\mathrm{Xu}$ Lisun, Zhao Dasu, etc systemize and file the work of The Longyinguan Music Score into The Meian Music Score after Wang Yanqing dies. In addition, they found the Meian Guqin Club and it is also a branch of Guqin of Zhucheng. [3] The style of Meian School is different from Wang Xinkui, but it still has obvious style of Shandong. Many outstanding talents of Guqin in "Meian Guqin " such as Liu Jingshao, Chen Xinyuan, Wu Zonghan, Shao Yuanfu, Gong Yi, Lin Youren, Li Yuxian, Liu Chicheng, Cheng Gongliang, Sun Keren and Liu Shanjiao, etc provide a wide range of examples for the research and development of the Zhucheng Guqin.

\section{B. The achievements acquired by Zhucheng Guqin in Shandong}

The inheritance and development of Guqin art have made rapid progress in recent years and been paid more and more attention. In August, 1977, in the "Traveler" 2 spaceship, the American deliberately placed a record and repeatedly played the world's most representative music works, including the melody of Guqin music Flowing Water played by the Chinese Guqin master Guan Pinghu. This melody of Guqin played for seven minutes is the master work of Zhucheng Guqin in Shandong, and it related to the good story created by the famous Guqin scholars Bo Ya and Zhong Ziqi jointly in the Spring and Autumn period. In 1999, the famous Guqin performer Gong Yi of our country moved musicians from all over the world by playing the Guqin in Vienna and let people around the world know the extensive and profound aspect of
Chinese Guqin. In November 7, 2003, the Zhucheng Guqin in Shandong was awarded "Human oral and intangible heritage master work" by UNESCO. The development of the Zhucheng Guqin in Shandong enters the glorious period and a large number of research groups are distributed throughout the country and many excellent talents in Guqin show business come into being.

\section{The problems exist in the development of Guqin of Zhucheng in Shandong}

- Zhucheng Guqin culture is lack of professional talents and gets a disordered education

Because the Guqing of Zhucheng suffered destruction in the period of the Great Cultural Revolution, and many Guqin scholars have passed away or get on in years and there are few successors of Guqin, and they are not very professional. So the inheritance dilemma is quite obvious. In view of the situation of a terrible shortage of talents, the development of Zhucheng Guqin has a long way to go. The Zhucheng Guqin is more different from other musical instruments and it has higher requirements for learner. It is also one of the reasons that the Zhucheng Guqin is lack of talents. The shortage of professional talents makes the education of Zhucheng Guqin disorder. Most of them teach Guqin in part-time or treat Guqin as sparetime study, which make the development of the Zhucheng Guqin is very slow. In the music grading test across the country, it is easy to see that professional Guqin talents are few and the professional talents in the local Zhucheng is fewer. So far, due to the rise of social culture, the social demand for music culture is growing and people's enthusiasm of studying Guqin runs high. But they cannot find the Guqin masters to learn Guqin and lack of right guidance and protection. Now these are severe obstructions in the development of the Zhucheng Guqin.

In the development of Guqin culture, many Guqin artists or fans make great efforts for the development of Guqin. They show the charm of Guqin culture and popularize Guqin art through the forms of community activity or concert.However, because people lack of understanding about Guqin even have some misunderstandings, there are some differences when they appreciate or use Guqin. The understanding for Guqin is not deep, let alone the mass communication of Guqin. Once lacking of the platform of mass communication, there won't be smooth channel for the development of Guqin. With the diversity of modern mass media, there should be various kinds of channels for people to know the Guqin art, such as radio, television, newspaper and network, etc. We should improve people's knowledge and understanding of Guqin and promote the spread and development of Guqin culture.

\section{Zhucheng Guqin culture is lack of continuable development}

The Guqin researchers think hard about what kind of development path that the inheritance and development of the Guqin culture of Zhucheng should go. The spread of Guqin art has always been a hands-on teaching and preaching. Inherent aesthetical standard and playing 
techniques endow Guqin art unique charm and it is also the charm of folk culture development in our country. For example, the influence of the "16 methods of tweedle" and "24 tones of tweedle" of Guqin playing have on Guqin art determines the style and aesthetic standards of Guqin art. And the principles of playing the Guqin such as "6-taboo and 7-no play" etc. is the unique temperament in Guqin playing. While in modern music field, the examples of the combination of Chinese and Western elements and making foreign things serve China are too numerous to mention one by one. To continue in the conservative or to change in the inheritance are the confusions in the development of Guqin and it is also the key that whether the Guqin art can be prosperous all the time. Sticking to the tradition of folk music, absorbing the essence of western music properly and then developing and inheriting it better, and handling the relationship between the development and inheritance are the basic principles of the inheritance of the art of Guqin culture and the protection of folk art. We should avoid inheriting stiffly and dogmatically and shouldn't make Guqin art in our country move towards vulgarity at random. The priority among priorities is reducing the commercial factors.

\section{The OBJECTIVE FACtors AfFecting the CUlturaL HERITAGE AND DEVELOPMENT OF ZHUCHENG GUQIN}

The development of Shandong Zhucheng Guqin also has been greatly impacted by the Western culture. In the aesthetic standards and playing skills of modern music, too many music artists accept the influence of Western music and change the musical style of Zhucheng Guqin into a nondescript marginal culture, which make Zhucheng Guqin culture develop towards a wrong way and distort the typical style and charm of Zhucheng Guqin art. This is a common problem in the development of many folk cultures.

The modernization of music education changes the musical aesthetic standard which is seriously westernized with the influence of Western culture. We extremely lack knowledge of national instruments and folk arts, as well as folk musical instruments. A lot of students have a little knowledge on Guqin, and Guqin teachers rarely make it easy for students to understand Guqin culture correctly. The westernized textbook and aesthetic standards make Guqin art difficult to gain a foothold in the music industry, resulting in the loss of a large number of students. At the same time, it also has a negative impact on the inheritance of Guqin art.

The development of Guqin art in Zhucheng has ever suffered several times of social changes. Especially during the Great Cultural Revolution, the Guqin culture was forced to be listed as "Feudalism, Capitalism and Revisionism" and was caught in the interrupt status. However, as the birthplace of the Guqin, Zhucheng still keeps a part of Guqin culture, and Zhucheng people love the Guqin culture. Therefore, it has become the living place of Zhucheng Guqin with local characteristics. The folk music, at the grassroots of musical culture, retains most of musical styles and instruments about Zhucheng Guqin. However, there is no doubt that during the Great Cultural Revolution the destruction of a large number of Guqin literature and ancient books and instruments has brought an irreparable loss to the inheritance of Zhucheng
Guqin. Thus, we will face a lot of difficulties in reexcavation and inheritance of Guqin culture. In addition, after the reform and opening up, Shandong Peninsula, as coastal open area, is located at the forefront position in the economical development and political reform. The impact of Western culture and the change of economic situation have greatly restricted the development of Guqin culture. The Guqin culture was fading inevitably. However, in Jiangsu and Zhejiang Provinces, Zhucheng Guqin branches are well protected with the influence of social change. The excavation and protection of Guqin culture have achieved some results, which is a gratification in the inheritance and development of Zhucheng Guqin art.

\section{THE INHERITANCE AND DEVELOPMENT STRATEGY OF ZHUCHENG GUQIN IN THE NEW ERA}

Although the Guqin culture has been listed as the world's intangible cultural heritage, but the inheritance and protection are limited by a variety of reasons in its development process, and its prospects still exist many difficulties. Especially in the future development process, it is an important work for the Guqin artists to protect and excavate the typical charm of Gunqin art, keep its unique cultural characteristics, and further carry it forward on this basis in order to highlight the inherent charm of traditional Chinese folk culture. According to the political environment and economic development model and people's spiritual and cultural quality under new situation, we need to protect the Guqin art through the following methods and strategies.

\section{A. To protect famous Guqin of past dynasties}

In the excavation and protection of folk culture, it is an important work to excavate and sort musical instruments. The Zhucheng Guqin culture has suffered a huge loss due to the destruction of famous Qin during the Great Cultural Revolution, which is irreparable. Therefore, it is extremely urgent to protect the world's rare remaining Guqin instruments. Once we lose them, it will no longer be able to recoup the loss. With the help of the current boom in the collection circle, we can advocacy Zhucheng Guqin and excavate and sort some Guqin instruments for the custody and protection.

\section{B. To collect and protect the audiovisual materials of the living Guqin masters}

It is an effective way to protect Guqin art's original style and artistic elements through collecting and sorting modern Guqin masters' audio-visual materials from folk and professional research societies, especially from the remaining few old men and folk artists. We can make their performance into audiovisual materials for safekeeping and preservation which will be valuable historical information for reference in the inheritance and development of its culture. 


\section{To collect and protect the audiovisual materials of elder Guqin masters}

We can collect and sort the audiovisual materials of famous Guqin artists before the Great Cultural Revolution to obtain more original historical materials about Guqin, for these materials are important cultural relics for the inheritance and development of Zhucheng Guqin and an important evidence of the intangible cultural heritage.

\section{To collect and sort Guqin masterpieces}

Folk bands and community associations and folk artists conserve the art of Zhucheng Guqin through traditional oral transmission and physical instruction. Through some of the folk artists are not highly educated, their brains are deposited with a lot of original Guqin songs. Once they are dead, some Guqin songs may be gone with them. Therefore, we can effectively excavate and protect the Zhucheng Guqin art by arranging special-designated personnel to collect and arrange these Guqin songs.

\section{E. To collect and sort Guqin literature}

It makes for the inheritance and development of Guqin and better research of the history of Guqin to collect and sort literature materials about Guqin through various channels, and thus protect our Guqin culture in a better manner.

\section{F. To develop and study modern Guqin music}

We can make a study after collecting and sorting Guqin literature, ancient books and musical materials, combined with modern public aesthetic taste in order to study and develop modern Guqin music that is consistent with aesthetic taste of the modern public and can fully highlight the art charm and playing style of Guqin art. It is a directional work to pass down and develop the Zhucheng Guqin culture. It can effectively protect the original charm and playing styles of Guqin culture and avoid malicious distortions under commercial interest by arranging specialized development and study, so that it can be avoided for classic Qin art to go the wrong way or loss its original artistic charm.

\section{G. To strengthen the legislative protection and management of the intangible cultural heritage}

The intangible cultural heritage is an important resource of our folk culture. It is called as non-material cultural heritage because of its importance on historical transmission and the urgency on protection and excavation. It is taken for granted to protect and utilize what our ancestors pass down to us. It is our responsibility to continue to pass down it rather than make it disappear in our hands. Therefore, we need to make laws and regulations in order to utilize and protect our intangible cultural heritage better. We can use the means of law to restrict and regulate the destruction of intangible cultural heritage and the malicious commercial benefit-seeking behavior by the use of intangible cultural heritage. It will provide legal basis for the inheritance and development of Zhucheng Guqin.

\section{H. To improve the harmonious society and cultural quality together}

The social root of the existence of Guqin art lies in the spiritual and cultural quality of the masses. The profitoriented, mercenary and impetuous and pompous attitude is not conducive to the survival and development of Zhucheng Guqin Art, for it is quiet, elegant, just and mild. The elegant art of Zhucheng Guqin may soon decline or disappear with too much fickleness and utility. Therefore, it is a foundation to create a harmonious social environment and improve people's cultural quality in order to create a harmonious, civilized and advanced society, and protect and develop the Guqin art. It is conducive to the health pursuit of people's spiritual life and the healthy development of Guqin culture by improving the cultural quality of the whole society.

\section{To guide the healthy development of the cultural societies}

Under rapid development of urban construction, cultural characteristics among regions gradually merge and regional cultures gradually disappear. All cultural societies confront with complicated factors in their development. The communication and cooperation of regional folk culture enter into a breaking-in period due the difference in region, cultural background and people's psychological quality, so that Zhucheng Guqin culture may be affected by other cultures. The establishment of community societies has provided us a platform to exchange modern culture. It is especially important to manage and supervise societies in this complex environment. All kinds of community activities appear under the impact of commercial interests, which needs us to guide and manage these societies by setting a correct guidance. It can effectively ensure the healthy development of community associations and improve the healthy development of Guqin art through the guidance of local governments and mass media.

\section{J. To gradually reform the quality education mode of primary and secondaryschools and strengthen folk music learning in primary and secondary schools}

From the childhood, we can attract people to love folk music and folk culture. The local governments can develop the quality education through folk culture with unique characteristics based on local folk culture to promote the inheritance and development of local folk culture.

\section{$K$. To strengthen the integrative development and artistic discussion of Zhucheng Guqin}

During more than 200 years of development course, Zhucheng Guqin has derived many sects and branches. Guqin societies in Zhucheng and Mei'an Qin societies and other societies in Jiangsu and Zhejiang should actively participate in the discussion and study of the development of Zhucheng Guqin, change their single-handed development situation and develop coordinately. 


\section{ACKNOWLEDGMENT}

We would like to thank all reviewers and editors for their valuable comments and suggestions during the review process.

\section{REFERENCES}

[1] Zhang Yujin. Shangdong Zhucheng Guqin. Jinan: Music Research, 1959.

[2] Xu Jian. First Edition of Qin History. Beijing: People's Music Publishing House, 1982.

[3] Liu Shanjiao and Mr. Wang Yanqing. Theory of Qin. Jinan: Music Research, 1996.

[4] Mi Yongying, Liu Shumei. Art Characteristics of Zhucheng Guqin and Its Development Status in Shandong. Qilu Realm of Arts, 2007, (3): 63-65. 